\title{
Simulation of heavy ion induced electron yield at grazing incidence
}

\author{
P. H. Stoltz* and S. Veitzer \\ Tech-X Corporation, 5621 Arapahoe Rd., Suite A, Boulder, Colorado 80303, USA \\ R. Cohen and A.W. Molvik \\ Lawrence Livermore National Laboratory, Livermore, California 94550, USA \\ J.-L. Vay \\ Lawrence Berkeley National Laboratory, Berkeley, California 94720, USA
}

(Received 22 June 2004; published 12 October 2004)

\begin{abstract}
We compare simulations to experiments measuring electron yield from heavy ions striking metal surfaces, in particular at grazing incidence, for $1.0 \mathrm{MeV}$ potassium and $182.0 \mathrm{MeV}$ gold ions striking stainless steel as measured in two recent experiments. We find the electron yield is proportional to within $3 \%$ for the potassium experiments and $13 \%$ for the gold experiments to the simulated energy deposited by the ions in a thin $(\approx 20 \AA)$ layer at the target surface. We discuss how nonequilibrium stopping and ion wake fields may account for the larger disagreement with the gold experiments. An analytic estimate based on specular reflection predicts the peak in simulated and measured electron yield as a function of angle to within a few percent.
\end{abstract}

DOI: 10.1103/PhysRevSTAB.7.103201

PACS numbers: 34.50.Dy, 52.20.Hv, 52.58.Hm

\section{INTRODUCTION}

Researchers believe the presence of stray electrons in ion accelerators limits the performance of many existing ion accelerators and may limit the performance of future ion accelerators [1]. One source of these electrons is the electrons induced by the ions scraping the beam pipe wall. These ion-wall collisions will be at grazing angles because the ion velocities are directed mostly along the pipe (in this paper, all angles are measured with respect to normal incidence). Recently, researchers have performed two experiments on grazing ion-wall collisions: one related to the Spallation Neutron Source (SNS) [2] performed at Brookhaven National Laboratory (BNL), and the other related to heavy-ion fusion (HIF) [3] performed at Lawrence Berkeley National Laboratory (LBL). Figure 1 shows measured electron yields [4] from the $\mathrm{LBL}$ experiment, where $1.0 \mathrm{MeV}$ potassium ions strike stainless steel. The solid line is the result predicted from a geometric theory (discussed below), and the figure shows that at the most grazing angles the measured yield deviates from this predicted yield.

The goal of this paper is to use simulation to improve understanding of electron generation from ion-wall interactions, with particular attention to grazing angles (angles are measured relative to normal). We first discuss the background necessary to understand this problem, in specific how the electron yield relates to the energy the ion deposits in the target, under what approximations the standard geometric angular scaling holds, and how the

\footnotetext{
*Corresponding author. Electronic address: pstoltz@txcorp.com
}

$1098-4402 / 04 / 7(10) / 103201(7) \$ 22.50$ repulsive force from the screened potential of the target ions suggests where to expect a peak in the yield as a function of angle. We then discuss the simulations, for which we used the SRIM code to model energy deposition as a function of angle in target surface layers of varying thickness. Finally, we compare the simulation results to experiments by converting the simulated energy deposition to an electron yield and finding the thickness which minimizes the difference between simulation and experiment. We compare simulations to the recent LBL experiments $(1.0 \mathrm{MeV}$ potassium ions hitting a stainless steel surface) and BNL experiments (182.0 MeV gold ions hitting a stainless steel surface). For the LBL experiments, we find the simulated energy deposited in surface layer of thickness $d_{\text {esc }} \approx 20 \AA$ predicts the electron yield as a function of angle to within 3\%. For the BNL experiments, the agreement is $13 \%$. We discuss how nonequilibrium stopping and ion wake fields could be responsible for the larger disagreement with the BNL experiments. We also compare simulations to measured electron yields for argon ions hitting a copper surface [5]. We find agreement to within roughly $3 \%$ using a $35 \AA$ simulation layer in those simulations, but the parameters for these experiments are in a range where assumptions we have made begin to break down. For all three experiments, an analytic model of reflection of the ion from the metal surface predicts to within a few percent the simulated and measured peaks in the electron yield.

\section{BACKGROUND}

In this section we review the theory of ion-induced electron yield. We discuss the relation between the energy 


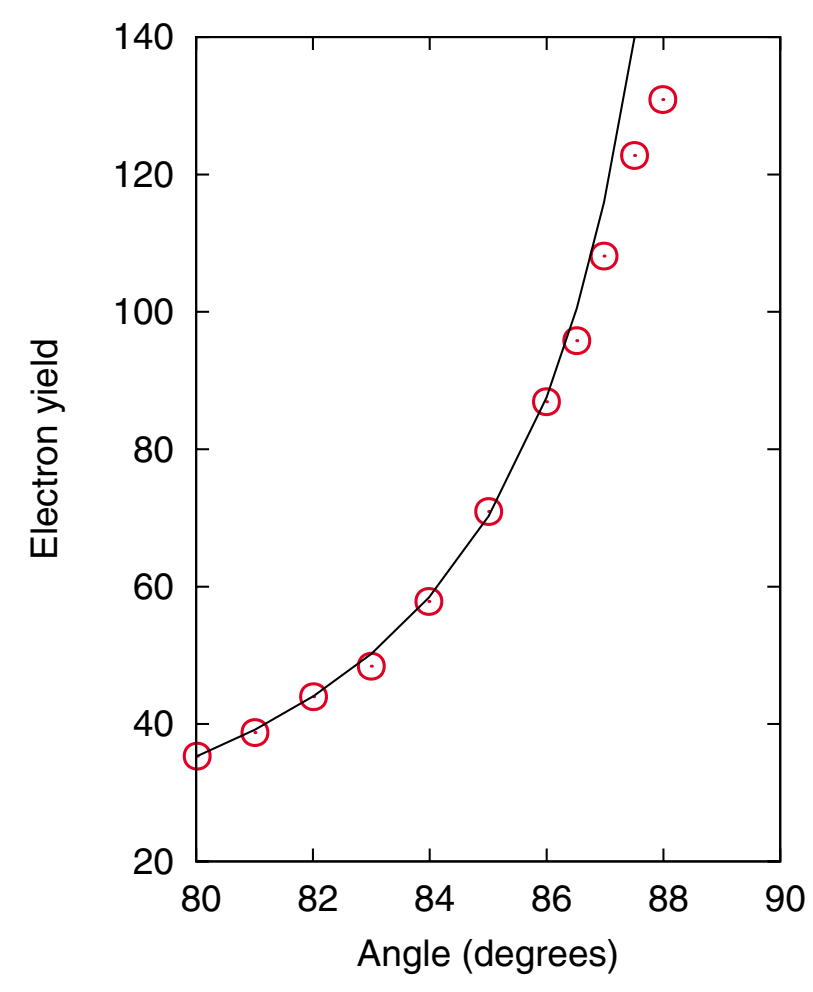

FIG. 1. (Color) Measured electron yield as a function of angle of incidence of the ion measured from the surface normal for 1.0 MeV potassium hitting stainless steel. The red circles are the electron yield as measured by researchers at LBL. The solid line is the prediction of a geometric theory discussed in Sec. II. One goal of this work is to better understand the deviation of the measured yield from the theory at grazing angles.

an ion deposits in a target and the subsequent electrons generated. We also discuss how an assumption of a thin layer for escaping electrons leads to an inverse cosine dependence of electron yield on incident ion angle (an angle of zero means normal incidence). We present a simple model for the ionization state of an ion as a function of velocity. Finally, we derive an approximate expression for the angle at which, for a given energy, the repulsive force of the target nuclei reflects the ion, and so one might expect a peak in the electron yield.

A basic model for ion-induced electron generation in a metal is to assume there is a layer at the surface of the metal from which electrons can escape. Electrons generated below that layer do not escape. One can estimate the number of electrons as the energy deposited by the ion into electrons in that surface layer divided by the energy required to generate one electron [6]. One typically multiplies this quantity by some factor representing the fraction of electrons moving toward the metal surface, giving the following expression for the electron yield:

$$
\gamma_{e}=P \frac{E_{\mathrm{dep}}}{E^{*}}
$$

where $\gamma_{e}$ is the electron yield, $E_{\mathrm{dep}}$ is the energy deposited by the ion into the electrons, $E^{*}$ is the energy required to generate one electron, and $P \approx 1 / 2$ is the fraction of electrons moving toward the metal surface. Equation (1) neglects any electrons produced by the ion colliding with target nuclei, and those nuclei recoiling and transferring energy into the electrons [7]. It also neglects any surface roughness effects [2]. For thin enough layers, one can replace the energy deposited by the path length times the electronic part of the energy lost per unit length:

$$
\gamma_{e}=\frac{P l}{E^{*}}\left(\frac{d E}{d x}\right)_{e}
$$

where $l$ is the path length the ion travels in the escape layer and $(d E / d x)_{e}$ is the electronic part of the energy loss per unit length. If scattering is neglected, the path length for a given angle of incidence is just the thickness of the escape zone divided by the cosine of the angle of incidence (where an angle of zero is normal to the surface). This gives

$$
\gamma_{e}=\frac{d_{\mathrm{esc}}}{\cos \theta} \frac{P}{E^{*}}\left(\frac{d E}{d x}\right)_{e},
$$

where $d_{\mathrm{esc}}$ is the thickness of the escape zone and $\theta$ is the angle of incidence with respect to the surface normal.

The quantities $d_{\mathrm{esc}}$ and $E^{*}$, to a large extent, are properties only of the target material and are independent of the incident ion. Typical values for a metal are $d_{\mathrm{esc}} \approx$ $10 \AA$ and $E^{*} \approx 25 \mathrm{eV}$, roughly of the order of an electron mean free path in metal and a few times a typical metallic work function. Some authors combine these together as $\Lambda=d_{\text {esc }} / E^{*}$. Reference [8] tabulates values of $\Lambda$ for many materials, and the typical values quoted above give $\Lambda=0.4$, which is within a factor of 2 of the value quoted copper, titanium, nickel, and aluminum. If one chooses $\Lambda$ so that Eq. (3) holds for a given target material with protons incident, then one can introduce $C$ to account for variations for ions other than protons:

$$
\gamma_{e}=C \Lambda \frac{P}{\cos \theta}\left(\frac{d E}{d x}\right)_{e}
$$

where $C=1$ by definition for protons, $C \approx 0.5$ for helium ions, and $C \approx 0.3$ for heavy ions [8].

An important quantity in the interaction of the ion and target is the projectile ionization state. The ionization state in which the ion is prepared is not necessarily the equilibrium ionization state with which it will move through the target. If the ion is moving at a high enough velocity, it will tend to lose electrons due to collisions with the target electrons. Conversely, if the ion is moving slowly, it will tend to gain electrons from the target. The important velocity to which to compare the ion velocity is a typical velocity of the electrons in the projectile. The Thomas-Fermi model predicts the electron velocities will be $v_{e} \approx v_{0} Z_{p}^{2 / 3}=\alpha c Z_{p}^{2 / 3}$, where $\alpha$ is the fine structure constant, $c$ is the speed of light, and $Z_{p}$ is the projectile 
atomic number. A model of the projectile equilibrium ionization state within the metal is

$$
Z=Z_{p}\left(1.0-e^{-0.95\left(v_{p} / v_{e}-0.07\right)}\right) .
$$

This neglects corrections due to the finite Fermi velocity of the target electrons [9], but is accurate for $v_{p} \gg v_{F}$, where $v_{F}$ is the Fermi velocity [10]. For $v_{p} \ll v_{F}$, one can replace $v_{p}$ by $0.75 v_{F}$. For $v_{p} \approx v_{F}$, Eq. (5) is more complicated [9]. The projectile ionization state is different from the effective charge, which includes a separate contribution from the target electrons penetrating the electron cloud of the projectile [11].

For glancing angles, where $\cos \theta \ll 1$, the inverse cosine behavior of Eq. (4) breaks down. If for no other reason, the path length $l$ of the incident ion eventually becomes of the order of the range of the particle. However, another effect that causes deviation from inverse cosine angular scaling is nuclear scattering. At grazing angles, particles can scatter out of the material before traversing the entire escape zone. One way to estimate the angle at which this becomes important is to calculate the angle at which the component of velocity of the ion toward the target is no longer large enough to overcome the repulsive potential of the target nuclei.

Using the Ziegler-Biersack-Littmark (ZBL) potential [9], the potential energy of an ion above an infinite twodimensional surface sheet of screened nuclei is

$$
\Phi_{\text {surf }}=2 \pi Z_{p} Z_{t} \frac{e^{2}}{a_{0}}\left(N^{1 / 3} a_{0}\right)^{2 / 3} \frac{a_{u}}{a_{0}} \sum \frac{a_{i}}{b_{i}} e^{-b_{i} z / a_{u}},
$$

where $z$ is the height above the surface, $e$ is the electronic charge, $a_{0}$ is the Bohr radius, $Z_{t}$ is the atomic number of the target nuclei, $N$ is the number density of the target, and $a_{u}$ is the ZBL universal screened atomic length [9]:

$$
a_{u}=\frac{0.8854 a_{0}}{Z_{p}^{0.23}+Z_{t}^{0.23}}
$$

The ZBL coefficients in the sum are $a_{1}=0.18, a_{2}=$ $0.51, a_{3}=0.28, a_{4}=0.03, b_{1}=3.20, b_{2}=0.94, b_{3}=$ 0.40 , and $b_{4}=0.20$. If the part of the kinetic energy associated with initial velocity of the ion toward the surface is lost to this potential energy, one can estimate the critical angle at which the ion kinetic energy is just enough to reach the surface with zero velocity toward the surface [12]:

$$
\frac{1}{2} M_{p} v^{2} \cos ^{2} \theta_{c}=2 \pi Z_{p} Z_{t} \frac{e^{2}}{a_{0}}\left(N^{1 / 3} a_{0}\right)^{2 / 3} \frac{a_{u}}{a_{0}} \sum \frac{a_{i}}{b_{i}},
$$

where $M_{p}$ is the projectile ion mass and $v$ is the (assumed nonrelativistic) projectile initial velocity. The angle, $\theta_{c}$, is given by

$$
\cos \theta_{c}=\sqrt{\frac{4 \pi Z_{p} Z_{t} e^{2}}{\beta^{2} M_{p} c^{2} a_{0}}\left(N^{1 / 3} a_{0}\right)^{2 / 3} \frac{a_{u}}{a_{0}} \sum \frac{a_{i}}{b_{i}}}
$$

As the incident angle approaches $\theta_{c}$, the ion deposits less energy in the target, and so $\theta_{c}$ gives an estimate of when the electron yield will have significantly deviated from inverse cosine. At angles significantly above $\theta_{c}$, the beam will not penetrate the target at all, and the electron yield could begin to decrease with increasing angle. Researchers have observed such peaks in the yield as a function of angle, and in Sec. IV we compare the location of simulated and experimental peaks with $\theta_{c}$. This expression for $\theta_{c}$ neglects the attractive force of the image charge [13] and the crystal structure of the target [14].

\section{SIMULATIONS}

The theory above gives a qualitative understanding of why the angular dependence of the electron yield is inverse cosine at moderate angles and at what angles one can expect significant deviation from inverse cosine dependence and perhaps even a peak in the yield as a function of angle. To understand in detail the electron yield at grazing angles, we performed simulations using the SRIM code [9].

The SRIM code is an ion transport code that calculates quantities like stopping and range of ions using a quantum mechanical treatment of ion-material collisions. SRIM models collisions between ions and target nuclei using the ZBL screened Coulomb potential, including electron shell effects and long-range interactions such as plasmons. SRIM uses an effective charge that includes a velocity dependent ionization state similar to Eq. (5) and a contribution due to target electrons.

The simulation technique we used to model electron yield is a technique first suggested by the authors of Ref. [4]. We assume Eq. (1) holds, and the electron yield is proportional to energy deposited. We used SRIM to estimate the energy deposited by the projectile in a surface layer of a target material for various angles of incidence. We then compare the simulation results for different surface layer thicknesses (representing $d_{\text {esc }}$ ) to see the effect of the thickness on the deposited energy as a function of angle. Figure 2 shows, as an example, results for $d_{\text {esc }}=10,20,30$, and $40 \AA$ for $1.0 \mathrm{MeV}$ potassium ions and a stainless steel target.

The limitations of the simulations are the assumption of an amorphous target, and so neglect structural properties of the material and any surface roughness. Further, the simulations are more valid for ion velocities that are nonrelativistic, but higher than the typical ion electron velocity (the velocity of a bound electron around the ion). 


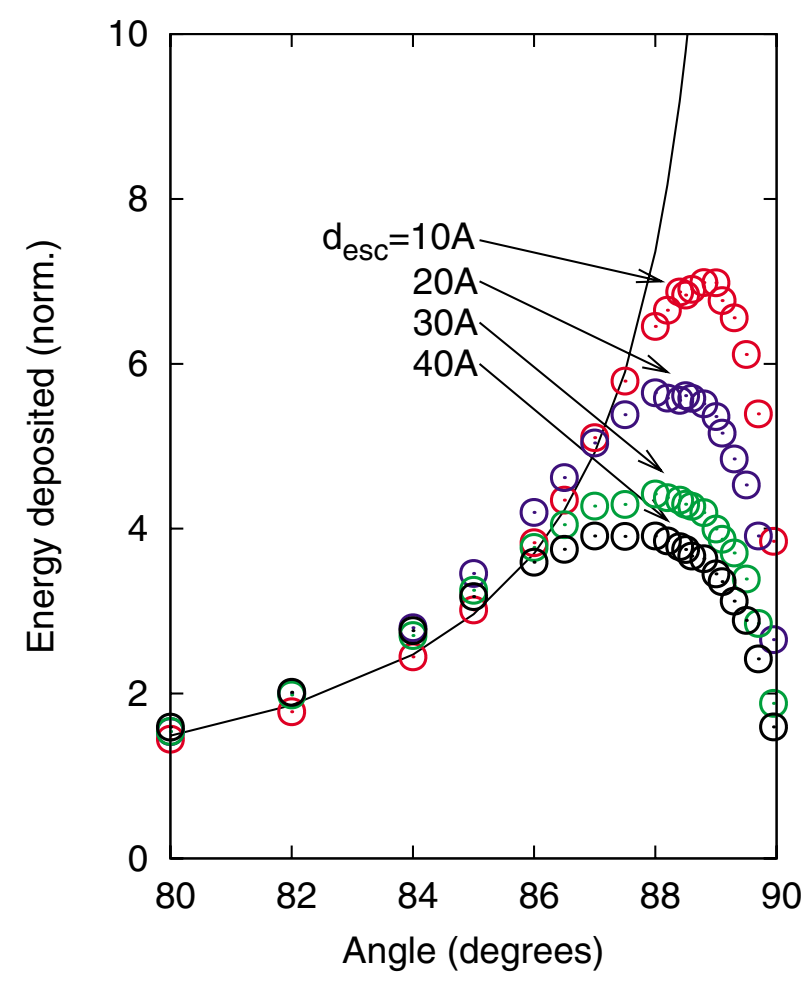

FIG. 2. (Color) Simulations of energy deposited as a function of angle of incidence of the ion measured from the surface normal for different thicknesses of target material. The deposited energy is normalized to the energy at the least grazing angle. Varying the thickness in the simulations allows one to see the effect of varying $d_{\text {esc }}$. These results are for $1.0 \mathrm{MeV}$ potassium hitting a stainless steel target.

\section{COMPARISON}

In this section we compare simulation results to experiments. We compare to the two recent experiments: one related to HIF and one related to the SNS. As a third comparison, we also compare to data from Ref. [5], though this experiment is for lower velocity ions, where the simulation results are more questionable. To compare the simulation to experiment, the simulated energy deposited is normalized to match the experimental results at the least grazing incidence [we also perform a consistency check using Eq. (4) to ensure this normalization is reasonable]. We then compare the simulated to measured electron yield for all other angles for different simulation thicknesses representing $d_{\mathrm{esc}}$ and find the thickness that minimizes the rms difference between the simulated and experiment results. Using the rms difference between simulation and experiment at all angles is a new approach not used in the original simulations using this technique [4]. We also compare the value of $\theta_{c}$ from Eq. (9) to the observed peaks in the simulations and experiments.

The first comparison is to the LBL experimental results [4]. In these experiments, singly ionized $1.0 \mathrm{MeV}$ potassium ions strike a stainless steel plate at angles ranging from $80^{\circ}$ to $88^{\circ}$. Figure 3 shows the measured (red circles) and simulated (blue squares) electron yields. The simulated electron yield is taken from simulations using a layer of $18 \AA$ and normalized to match the data at the least grazing angle ( 35.3 at $80^{\circ}$ in this case). The solid line is inverse cosine scaling, also normalized to match the data at the least grazing angle. The rms difference between data and simulated values is $3 \%$, and this was the minimum for the different simulated thicknesses we tried. Also, using Eq. (9), $\theta_{c} \approx 87.9^{\circ}$ for these parameters. The LBL researchers did not observe a peak in the data, but their most grazing angle was $88.0^{\circ}$. We observed a peak in the simulations at roughly $88.2^{\circ}$, within a percent of the predicted $\theta_{c}$.

We perform four consistency checks: we check that (i) the electronic part of the stopping power is larger than the nuclear part, (ii) the equilibrium ionization state is near the initial experimental ionization state, (iii) the range is larger than the geometric path through $d_{\mathrm{esc}}$ for an ion at the most grazing angle, and (iv) the yield

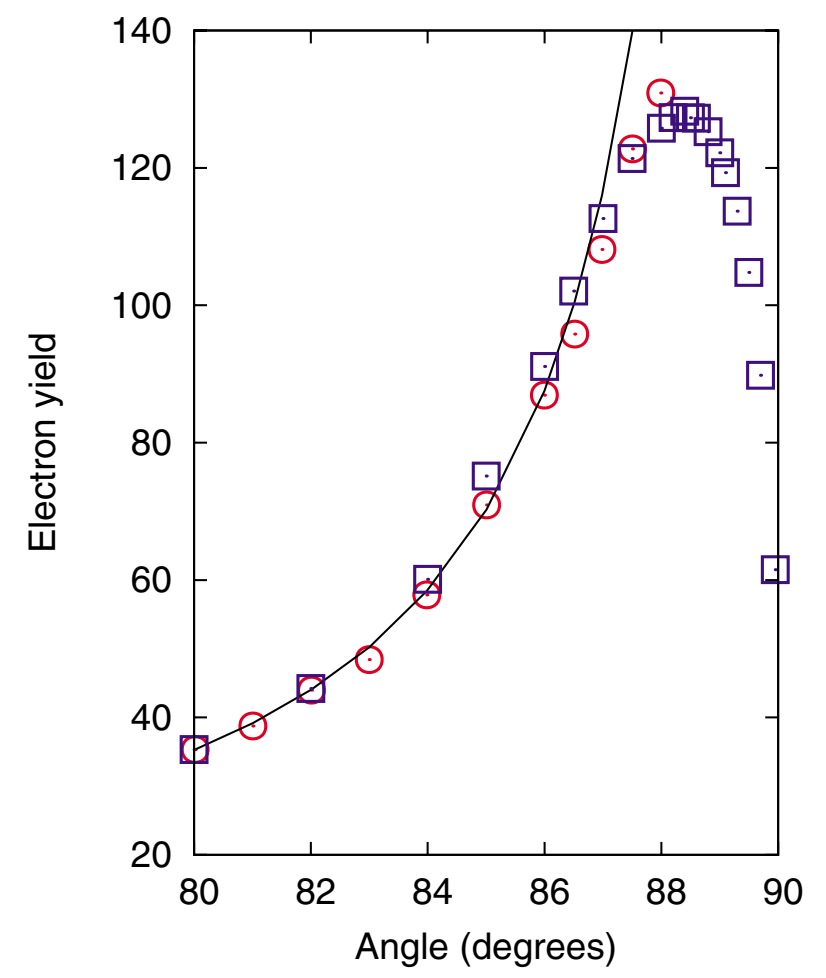

FIG. 3. (Color) The measured (red circles) and simulated (blue squares) electron yields as a function of angle of incidence of the ion measured from the surface normal for $1.0 \mathrm{MeV}$ potassium hitting stainless steel. The simulations used $d_{\text {esc }}=18 \AA$. The simulation values are normalized to match the measured yield at the least grazing angle $\left(80^{\circ}\right.$ in this case). The rms difference between simulated and measured yield is $3 \%$. Equation (9) gives $\theta_{c} \approx 87.9^{\circ}$ for these parameters. The LBL researchers did not observe a peak in the data, but their most grazing angle was $88.0^{\circ}$. We observed a peak in the simulations at roughly $88.2^{\circ}$. The solid line is inverse cosine scaling. 
predicted by Eq. (4) is close to the measured yield. The first check ensures the validity of assuming the contribution to the electron yield by recoiling target nuclei is negligible. At $1.0 \mathrm{MeV}$, SRIM predicts the electronic part of the stopping power for a potassium ion hitting stainless steel is $205.5 \mathrm{eV} / \AA$, roughly 5 times larger than the nuclear part. The second check ensures the simulations will be in a regime near what the experiments measured. The equilibrium ionization state of these ions is approximately 1.6, using Eq. (5), close to the 1.0 ionization state used in experiment. The third check determines to what degree the stopping power is remaining dominantly electronic, since at the end of the path of the ion, the stopping is dominantly nuclear. The range is $0.4 \mu \mathrm{m}$. To travel half the range while within a distance $d_{\text {esc }}$ of the surface, the ion would need to enter at $89.5^{\circ}$, which is beyond the experiments and simulations. The fourth check ensures the rescaling of the energy deposited to an electron yield is reasonable. Using typical values of $\Lambda=0.32 \AA / \mathrm{eV}$, $P=0.45$, and $C=0.3$, we predict, using Eq. (4), an electron yield of $\gamma_{e} \approx 49.7$ at $\theta=80.0^{\circ}$, accurate to within $40 \%$ of the measured value.

The second comparison is to experimental results from BNL performed with relevance to the SNS [2]. These researchers measured electron yield for protons, oxygen ions, and gold ions in differing ionization states striking stainless steel at angles from $0^{\circ}$ to over $89.9^{\circ}$. We chose to compare with the data for gold ions $(182.0 \mathrm{MeV}$ and an initial ionization state of 31) because that data showed a peak in the yield. Figure 4 shows the measured (red circles) and simulated (blue squares) electron yields. The simulated electron yield is taken from simulations using a $20 \AA$ layer, but in this case normalized to match Eq. (4) rather than experiment at normal incidence because of the difference between the initial experimental ionization state and the equilibrium ionization state (we discuss this below in more detail). The value $d_{\mathrm{esc}}=20 \AA$ is within $10 \%$ of the value used above for the comparisons with LBL data, consistent with the idea that $d_{\text {esc }}$ is largely a target material parameter, independent of the projectile ion. The solid line is inverse cosine scaling, also normalized to match the data at the least grazing angle. The rms difference between data and simulated values is $13 \%$. The peak in the experimental results occurs at $89.92^{\circ}$. For the BNL parameters, $\theta_{c} \approx 89.7^{\circ}$, within a percent of the measured value. The peak in the simulations occurs at $89.75^{\circ}$. The authors of Ref. [2] calculated an angle of $88.5^{\circ}$ using the formula in Ref. [5], which attempts to account for the crystal structure of the target. The authors of Ref. [2] also suggest multiple scattering as opposed to reflection as a mechanism for this peak, though their calculation is not quantitative.

We again performed our four consistency checks. At an energy of $182.0 \mathrm{MeV}$, SRIM predicts the electronic part of the stopping power is $4296.0 \mathrm{eV} / \AA, 100$ times larger than

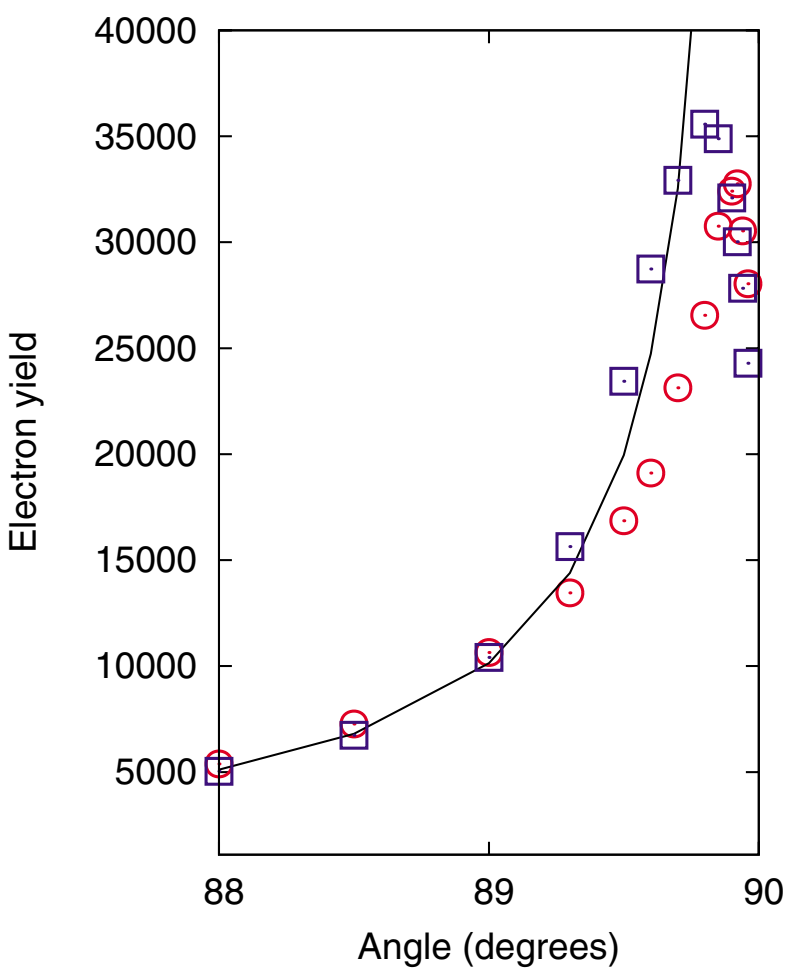

FIG. 4. (Color) The measured (red circles) and simulated (blue squares) electron yields as a function of angle of incidence of the ion measured from the surface normal for $182.0 \mathrm{MeV}$ gold hitting stainless steel. The simulations used $d_{\mathrm{esc}}=18 \AA$. The simulation values are normalized to match Eq. (4) at $0^{\circ}$. The rms difference between simulated and measured yield is $13 \%$. Equation (9) gives $\theta_{c} \approx 89.7^{\circ}$ for the BNL parameters. The peak in the experimental results occurs at $89.92^{\circ}$. The peak in the simulations occurs at $89.75^{\circ}$. The solid line is inverse cosine scaling.

the nuclear part. The equilibrium ionization state of these ions is approximately 17.4 , using Eq. (5), which is significantly different from the ionization state of 31 used in the experiment (we discuss this below). The range is $8.5 \mu \mathrm{m}$. To travel half the range while within a distance $d_{\text {esc }}$ of the surface, the ion would need to enter at $89.98^{\circ}$, which is beyond the experiments and simulations. Also, using typical values of $\Lambda=0.32, P=0.45$, and $C=0.3$, we get, using Eq. (4), a predicted electron yield of $\gamma_{e} \approx$ 180.4 at $\theta=0$, accurate to within $14 \%$ of the measured value (we also discuss this below).

Two factors make the comparisons with the BNL simulations difficult: (i) the initial ionization state of the ion is significantly different from the equilibrium state [8], and (ii) the projectile atomic number is larger than other experiments considered and is in a regime where effects such as ion wake fields may be significant [15]. We did not consider these effects for the LBL experiment because (i) the difference between the initial and equilibrium ionization state was less than one, and (ii) the projectile atomic number was small, meaning a 
smaller wake field. Quantifying the effects of these two factors is difficult, but qualitatively one can argue that the simulated yield will be lower than the experimental yield at angles near normal because the ionization state used in the simulations is the equilibrium state and is less than the experimental ionization state of 31 . For near normal angles, the ion may not have time to reach its equilibrium value before passing through the emission layer. This is consistent with the fact that the estimated yield at normal incidence was $\gamma_{e} \approx 180.4$, which used the equilibrium stopping power, was lower than the experimental yield of $\gamma_{e}=209$, and is the reason we normalized the simulations to 180.4 at $\theta=0$.

A second qualitative argument is that the experimental yield for grazing incidence will be lower than the simulated yield for heavier ions such as gold because the attractive wake field of the ion significantly modifies the electron dynamics. The authors of Ref. [15] suggest that at moderate energies $(\sim 5.0 \mathrm{MeV} / \mathrm{amu})$ the reduction can be as large as an order of magnitude, although other researchers were unable to verify those results [16]. Qualitatively, however, a reduction in yield due to the attractive ion wake field is consistent with the experimental yield being lower than the simulated yield at grazing angles, as seen in Fig. 4.

The third comparison is to experimental results of Ref. [5]. These experiments measured electron yields for seven different ions (all singly ionized) striking a copper surface. We chose to compare to the data for $0.04 \mathrm{MeV}$ argon ions, since it showed a peak in the yield. Figure 5 shows the measured (red circles) and simulated (blue squares) electron yields. The simulated electron yield is taken from simulations using a $35 \AA$ layer and normalized to match the data at the least grazing angle $\left(2.0\right.$ at $0^{\circ}$ in this case). The rms difference between data and simulated values is $3.0 \%$. The measured peak in the yield and the simulated peak are at $\theta \approx 83.0^{\circ}$. For the parameters of these experiments, $\theta_{c} \approx 78.9^{\circ}$, roughly $5.0 \%$ too low. The authors of Ref. [5] predicted a peak at $\theta=80.6^{\circ}$ using a method similar to the technique used to obtain Eq. (9), but that included effects of the crystal structure of copper.

At $0.040 \mathrm{MeV}$, SRIM predicts the electronic part of the stopping power is $32.1 \mathrm{eV} / \AA$, roughly 0.26 times the nuclear part. At this energy, the ion velocity is well below the Fermi velocity of the copper electrons, so Eq. (5) requires replacing $v_{p}$ by $0.75 v_{F}$ [9], and this yields an equilibrium ionization state of 0.2 . Whether this is close enough to the experimental initial ionization state of one is somewhat beside the point, since for these parameters the target Fermi electrons are going to dominate the effective charge and the electronic stopping power. The range of the argon is $0.018 \mu \mathrm{m}$, and so an ion entering at $67.1^{\circ}$ would travel half its range within the escape layer. However, this is again almost beside the point in this case,

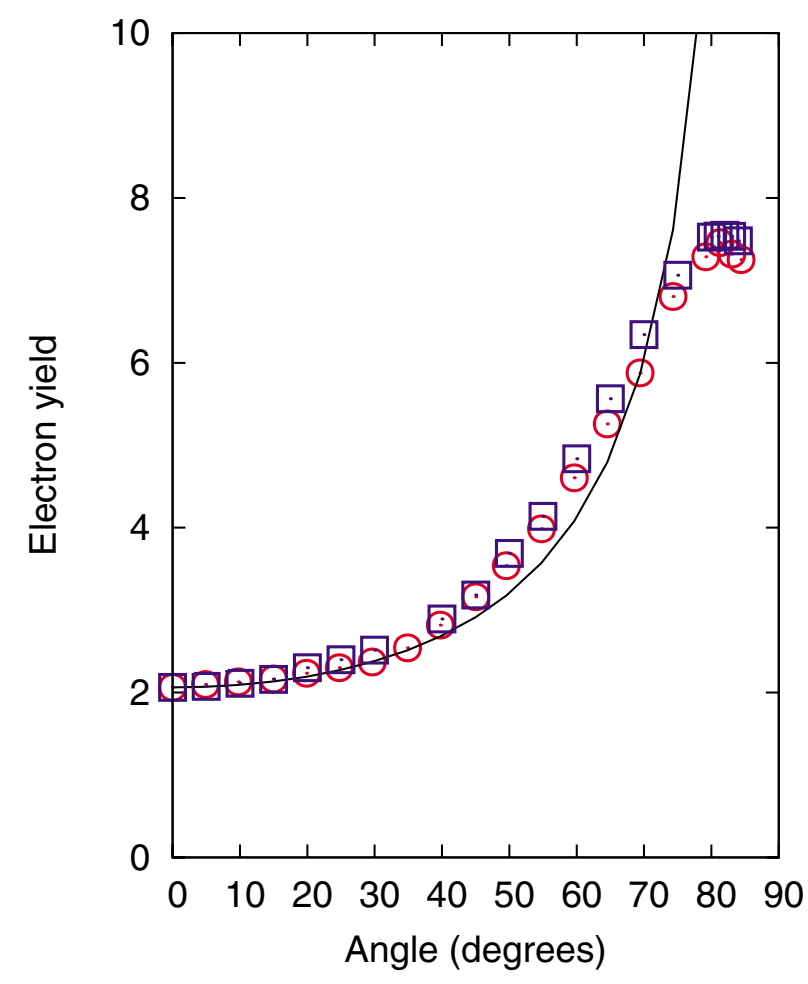

FIG. 5. (Color) The measured (red circles) and simulated (blue squares) electron yields as a function of angle of incidence of the ion measured from the surface normal for $0.04 \mathrm{MeV}$ argon hitting copper. The simulations used $d_{\mathrm{esc}}=35 \AA$. The simulation values are normalized to match the measured yield at the least grazing angle $\left(0^{\circ}\right.$ in this case). The rms difference between simulated and measured yield is $3.0 \%$. Equation (9) gives $\theta_{c} \approx 78.9^{\circ}$ for these parameters. The peak in the experimental and simulation results occurs at $83.0^{\circ}$. The solid line is inverse cosine scaling.

since the ion stopping is already dominantly nuclear. Finally, using typical values of $\Lambda=0.32, P=0.45$, and $C=0.3$, we get, using Eq. (4), a predicted electron yield of $\gamma_{e} \approx 1.3$ at $\theta=0$, accurate to within $35 \%$ of the measured value.

Despite the agreement to within a few percent as shown in Fig. 5, we have the least understanding of and confidence in this comparison. The experimental parameters are in a regime where the approximations we have made begin to break down. Most notably, the nuclear part of the energy loss is larger than the electronic part. Consequently, neglecting the electrons produced by recoiling target nuclei may not be justified. This is consistent with the fact that the predicted yield at normal incidence is lower than the measured yield by $35 \%$. Also, the ion velocity $(\beta=v / c \approx 0.0015)$ is lower than both the ion electron velocity $(\beta \approx 0.05)$ and the target electron Fermi velocity ( $\beta \approx 0.005)$, which is a regime where many of the SRIM algorithms are least reliable. However, we show these results because the agreement to within a few percent seemed worth noting for possible future explanation. 


\section{CONCLUSION}

The goal of this paper was to use simulation to improve understanding of electron generation from ion-wall interactions, with particular attention to grazing angles. We discussed how the electron yield relates to the energy the ion deposits in the target, under what approximations the standard inverse cosine angular scaling holds, and how the repulsive force from the screened potential of the target ions can create a peak in the yield as a function of angle. We discussed the simulations, for which we used the SRIM code to model energy deposition as a function of angle in target surface layers of varying thickness. We compared the simulation results to the recent experiments from LBL and BNL, where we find the simulated energy deposited in a stainless steel surface layer of $\approx 20 \AA$ predicts the electron yield as a function of angle to within $3.0 \%$ for the LBL experiment and within $13 \%$ for the BNL experiment. We discussed how nonequilibrium stopping and ion wake fields could account for the larger error in the BNL comparisons. Finally, we also compared simulations to measured electron yields for argon ions hitting a copper surface, where we find $3.0 \%$ agreement using a $35 \AA$ thick surface layer. We do not have a full understanding of the agreement for this case, as many approximations we have made break down in that parameter regime. For all three experiments, an analytic model of reflection of the ion from the metal surface predicts to within a few percent the simulated and measured peaks in the electron yield.

\section{ACKNOWLEDGMENTS}

This work was supported partially by the U.S. Department of Energy by Small Business Innovation Research Grant No. DE-FG02-03ER83797. This work was performed under the auspices of the U.S.
Department of Energy by Lawrence Livermore National Laboratory under Contract No. W7405-ENG-48 and by Lawrence Berkeley National Laboratory under Contract No. DE-AC03-76F00098.

[1] M. Blaskiewicz, M. A. Furman, M. Pivi, and R. J. Macek, Phys. Rev. ST Accel. Beams 6, 014203 (2003).

[2] P. Thieberger, A. L. Hanson, D. B. Steski, V. Zajic, S. Y. Zhang, and H. Ludewig, Phys. Rev. A 61, 042901 (2000).

[3] P. A. Seidl, in Proceedings of the 14th International Symposium on Heavy Ion Inertial Fusion, Moscow, 2002 (Institute of Experimental and Theoretical Physics, Moscow, 2002).

[4] A. W. Molvik, M. K. Covo, F. M. Bieniosek, L. Prost, P. A. Seidl, D. Baca, A. Coorey, and A. Sakumi, Phys. Rev. ST Accel. Beams 7, 093202 (2004).

[5] B. Svensson, G. Holmen, and A. Buren, Phys. Rev. B 24, 3749 (1981).

[6] E. Sternglass, Phys. Rev. 108, 1 (1957).

[7] J. Schou, Phys. Rev. B 22, 2141 (1980).

[8] H. Rothard, K. Kroneberger, A. Clouvas, E. Veje, P. Lorenzen, N. Keller, J. Kemmler, W. Mechbach, and K.-O. Groeneveld, Phys. Rev. A 41, 2521 (1990).

[9] J. F. Ziegler, J. P. Biersack, and U. Littmark, The Stopping and Range of Ions in Solids (Pergamon, New York, 1985).

[10] N.W. Ashcroft and N. D. Mermin, Solid State Physics (Saunders, Orlando, FL, 1976).

[11] W. Brandt and M. Kitagawa, Phys. Rev. B 25, 5631 (1982).

[12] M. J. Witcomb, J. Appl. Phys. 48, 434 (1977).

[13] J. I. Juaristi, F. J. Garciade Abajo, and P. M. Echenique, Phys. Rev. B 53, 13839 (1996).

[14] M. J. Witcomb, J. Mater. Sci. 9, 1227 (1974).

[15] J. E. Borovsky and D. M. Suszcynsky, Phys. Rev. A 43, 1433 (1991).

[16] H. Rothard, Nucl. Instrum. Methods Phys. Res., Sect. B 10, 1 (1998). 